\title{
Impact of COVID-19 lockdown on sleep quality in university students and administration staff
}

\author{
Sara Marelli ${ }^{1,2} \cdot$ Alessandra Castelnuovo $^{1} \cdot$ Antonella Somma $^{2} \cdot$ Vincenza Castronovo $^{1} \cdot$ Samantha Mombelli $^{1}$. \\ Daniela Bottoni ${ }^{1} \cdot$ Caterina Leitner $^{1} \cdot$ Andrea Fossati $^{2} \cdot$ Luigi Ferini-Strambi $^{1,2} \mathbb{C}$
}

Received: 23 June 2020 / Revised: 3 July 2020 / Accepted: 4 July 2020 / Published online: 11 July 2020

(c) Springer-Verlag GmbH Germany, part of Springer Nature 2020

\begin{abstract}
Introduction In Italy, lockdown due to COVID-19 health emergency started on March 10 and partially ended on May 3rd, 2020. There was a significant increase of psychological distress and symptoms of mental illness, and worsening of quality of sleep in the general population.

Methods Participants completed an anonymous web-based survey that include questionnaires about sleep and anxiety and depression symptoms. Our sample included 400 subjects: 307 students (mean age $22.84 \pm 2.68$ ) and 93 university administration staff workers (mean age $37.02 \pm 12.46$ ).

Results we found an increase in Bed Time hour, Sleep Latency, and Wake-Up time between before and during COVID-19 emergency and a worsening of sleep quality and of insomnia symptoms. In particular, during the lockdown, the impact of the delay in Bed Time and in Wake-Up was more pronounced in students. In workers, we observed a prevalence of maintenance insomnia before COVID-19 of 24\% that significantly increase during COVID-19 reaching 40\%, while workers with difficulties in sleep initiation were only $15 \%$ that increased to $42 \%$. In our sample, $27.8 \%$ showed depressive symptoms, while $34.3 \%$ showed anxious symptoms, in particular in students.

Conclusion The impact of lockdown was greater in students than in workers, and in females than in males. Concerning the psycho-emotional aspects, about one-third of our sample showed depressive or anxious symptoms. The results of our study may provide support for the implementation of some interventions for well-being in pandemic condition.
\end{abstract}

Keywords COVID-19 $\cdot$ Sleep $\cdot$ Depression $\cdot$ Anxiety

\section{Introduction}

In Italy, lockdown due to Corona Virus Disease 2019 (COVID-19 known as 2019-nCoV) health emergency started on March 10 and partially ended on May 3rd, 2020. This decision, as in many other countries, allowed reduced use of healthcare system and limited the viral transmission [1]. The Italian population was placed in social isolation for almost 2 months with the only permission to leave home for buying food or medicine. These restrictions greatly changed

Luigi Ferini-Strambi

ferinistrambi.luigi@hsr.it

1 Department of Clinical Neurosciences, Neurology-Sleep Disorders Center, IRCCS San Raffaele Scientific Institute, Milan, Italy

2 Faculty of Psychology, "Vita-Salute" San Raffaele University, Milan, Italy lifestyles and social relationships creating in many individuals increased levels of anxiety also due to the fear of being infected. There was a significant increase of psychological distress and symptoms of mental illness in the general population [2]. Even if the restriction of freedom was fundamental to reduce the virus diffusion, a possible high psychological cost was induced by COVID-19 epidemic.

Previous studies found that acute infectious disease, like SARS, can cause anxiety, depression, stress, and posttraumatic stress disorder both in survivors and non-infected people [3-5]. Previous studies showed a negative impact on mental health on people that face sudden events that could even culminate in similar post-traumatic stress disorders symptomatology [6]. Traumatic events such as those caused by COVID-19 outbreak can produce psychological distress and anxiety symptoms which negatively impact sleep quality [7]. 
For this reason, researchers focused on physical and mental health, and sleep during COVID-19 emergency. Recent Chinese studies on the general population found that female gender, being student, having suggestive COVID-19 symptoms, and perceived poor quality of life were associated with higher rate of anxiety and depression [8-11]. Another study showed that PTSD symptoms were reported by $7 \%$ of Wuhan residents after the COVID-19 outbreak, in particular by women. People younger than 35 years old and that spend more than $3 \mathrm{~h}$ following COVID-19 news showed higher levels of anxiety [12].

A recent review on sleep problems during home confinement due to COVID-19 outbreak by European CBT-I Academy focused the attention on sleep and insomnia [13]. Cellini et al. [14] assessed changes in sleep pattern, sense of time, and use of electronical devices in 1310 young adults (workers and university students), and they found an increase of digital media's use in the evening before bedtime. Moreover, they found that people went to bed and woke up later, and spent more time in bed, with a lower quality of sleep. Participants with higher levels of depression, anxiety, and stress showed more sleep problems. A study conducted by Li et al. [15] showed that the prevalence of insomnia increased significantly during the COVID-19 outbreak (in some cases new onsets of insomnia), that time in bed (TIB) and total sleep time (TST) increased, and that sleep efficiency significantly decreased.

The aim of this study was to assess the psychological impact of COVID-19 emergency period in a sample of administration staff and students of the University VitaSalute San Raffaele in Milan, Italy. In particular, we focused on perceived sleep quality and psycho-emotional well-being (anxiety and depression symptoms).

\section{Materials and methods}

\section{Participants}

Participants completed an anonymous web-based survey from March 24 to May 3, 2020 after agreeing to an electronic informed consent requested for each participant. The survey took $20 \mathrm{~min}$ to be completed.

A total of 400 participants recruited in the University Vita-Salute San Raffaele, Milan (students and administration staff) with a mean age of $22.84 \pm 2.68$ (range 19-67 yrs) completed the survey. None of the participants reported symptoms of COVID-19.

\section{Measures}

\section{Sleep questionnaires}

All participants completed the Italian versions of the Pittsburgh Sleep Quality Index (PSQI) [16, 17], the Insomnia
Severity Index (ISI) [18, 19], and the Morningness-Eveningness Questionnaire [20, 21].

PSQI is a self-administered questionnaire that assesses the quality of sleep with questions on the last month. Scores higher than five indicate poor sleep. From the PSQI, we took information about how many hours participants spent in bed (TIB; min) and sleep (TST; min), at what time they went to bed to sleep (BT; hr), and at what time they woke up in the morning (WU; hr).

ISI shows the impact of insomnia. The total score ranges from 0 to 28, with higher score indicating greater insomnia severity. Each item is rated on a 5-point Likert scale of 0-4 (for items $1-3,0=$ no problem, $4=$ very severe problem; for item $4,0=$ very satisfied, $4=$ very dissatisfied; for items $5-7$, $0=$ not at all, $4=$ very much).

To evaluate the different types of insomnia, it is possible to look at the first three items: Item 1 refers to sleep initiation, item 2 refers to sleep maintenance, and item 3 refers to early morning awakening. A cut-off value of 15 has been used as threshold for a clinically relevant insomnia. The total score of the questionnaire is divided as follows: $0-7$, no significant insomnia; 8-14 subthreshold insomnia; 15-21, moderate insomnia and 22-28 severe insomnia [18].

MEQ is a self-administered questionnaire that assesses chronotype [20, 21]. Using the 19 MEQ items, we can obtain a minimum score of 16 and a maximum score of 86 . This questionnaire shows five categories (cut-offs 16-30 "definitely evening types", 31-41 "moderately evening types", 42-58 "intermediate types", 59-69 "moderately morning types", 70-86 "definitely morning types") [20]. For our analysis on chronotype, we divided the categories in three subgroups to make the sample more homogeneous (cut-offs below 42 "evening types", 42-58 "intermediate types", above 58 "morning types") [22].

\section{Anxiety and depression symptoms questionnaires}

All participants completed the Italian versions of Beck Anxiety Inventory (BAI) $[23,24]$ and Beck Depression Inventory-II (BDI-II) [25, 26].

BAI is a self-administered questionnaire that evaluates the severity of symptoms of anxiety. It is composed by 21 items on a four-point Likert scale. The scores are minimal anxiety (0-7), mild anxiety (8-15), moderate anxiety (16-25), and severe anxiety (30-63). Anxiety and depressive disorders are strongly related to each other. For this reason, the BAI correlates highly with the BDI-II, indicating that they can be useful for the clinical evaluation.

BDI-II is a self-administered questionnaire, composed by 21 items on a four-point Likert scale, that assesses depressive symptoms. The scores are divided in two subscales: affective symptoms (eight items) and somatic symptoms (13 
items). Total score of $0-13$ is considered minimal range, 14-19 is mild, 20-28 is moderate, and 29-63 is severe [25].

\section{Statistical analysis}

Statistical analyses were performed using SPSS 22 and Matlab R2016b. The significance of multivariate mean comparisons on sleep variables pre- and during-COVID-19 was tested by computing paired-sample Hotelling $T^{2}$ test, followed by Bonferroni paired-sample $t$ tests. One-way MANOVA was used to test the multivariate effect of chronotype on COVID-19-related sleep variables; Pillai $V$ statistic was used as multivariate omnibus test. Bonferroni-protected $F$ tests were computed to evaluate the significance of the chronotype effect on the individual sleep variables. In the case of significant chronotype effects on sleep variables, the possible effect of chronotype on the change in sleep variables from pre- to during-COVID-19 was tested using repeated-measure ANOVA. Moreover, ANOVA was performed to assess the differences between chronotypes preand during-COVID-19. One-way MANCOVA was used to assess the significance of the multivariate effect of employment (i.e., student vs. worker) on sleep variables while controlling for age effects. Pillai $V$ statistic was used as multivariate omnibus significance test. In the case of significant Pillai $V$ value, Bonferroni-protected $F$ tests were computed. The presence of a significant difference in mean age between students and workers was previously tested by computing independent-sample $t$ test. The possible association between participant's age, sleep variables, and scores on anxiety and depression was tested by computing Pearson $r$ coefficients. Moreover, we assessed gender differences from pre- to during-COVID-19 in students and workers. The Chi-square test was used to test the significance of the association between nominal variables.

\section{Results}

Our sample included 400 subjects: 307 students (mean age $22.84 \pm 2.68$ ) and 93 university administration staff (workers) (mean age 37.02 \pm 12.46$)[t(94.600)=-10.890$; $p<0.001] .75 .8 \%$ were females and the distribution of gender was similar in workers $(78.5 \%$ females $)$ and students (74.9\% females).

When we analyzed subjective data about sleep, we found an increase in BT hour, SL, and WU time between before and during COVID-19 emergency. There were no differences in TST and in TIB. Moreover, we observed a worsening of sleep quality (PSQI total score) and of insomnia symptoms (ISI total score) (Table 1).

Paired-sample multivariate omnibus test was highly significant $\left[T^{2}=270.23, F(7393)=38.02, p<0.001\right]$. In our sample, $25.5 \%$ was "evening types", 54\% "intermediate types", and $20.5 \%$ "morning types". A multivariate significant effect of chronotype was observed on dependent variables [pre-COVID-19, Pillai $V=0.338 ; p<0.001$, [during-COVID-19, Pillai $V=0.387 ; p<0.001$ ]. Concerning univariate $F$ tests on pre-COVID-19, chronotype showed an effect on BT $[F(2330)=53.857 ; p<0.001 ; \eta 2=0.246]$, $\mathrm{SL}[F(2330)=10.214 ; p<0.001 ; \eta 2=0.058]$, WU $[F$ $(2330)=39.862 ; p<0.001 ; \eta 2=0.195]$, and PSQI total score $[F(2330)=5.655 ; p=0.004 ; \eta 2=0.033]$. Concerning univariate $F$ tests on during-COVID-19, chronotype showed an effect on BT $\left[F(2377)=76.826 ; p<0.001 ; \eta^{2}=0.290\right]$, WU $\left[F(2377)=72.826 ; p<0.001 ; \eta^{2}=0.279\right]$.

We evaluated the influence of chronotype and we found a statistically significant effect between pre- and duringCOVID-19 in all sleep parameters except TST, but these effects remain significant after Bonferroni correction only in BT $[F(1344)=88.483 ; p<0.001]$ and WU $[F$ $(1343)=145.644 ; p<0.001]$.

According to Dunn-Bonferroni contrasts, we found on pre-COVID-19 that "evening types" showed a higher BT than "intermediate types" and "morning types" [24:33 $\pm 00: 06$ vs $23: 34 \pm 00: 04$ vs $22: 58 \pm 00: 07 ; p<0.001]$, and a higher WU $[08: 36 \pm 00: 07$ vs 07:44 \pm 00:04 vs 06:56 $\pm 00: 08 ; p<0.001]$. According to Dunn-Bonferroni contrasts, we found on during-COVID-19 that "evening types" showed a higher BT than "intermediate types" and "morning types" [01:32 \pm 00:08 vs 23:57 \pm 00:05 vs 23:19 $\pm 00: 08 ; p<0.001]$, and a higher WU [10:00 $\pm 00: 06$ vs 08:39 $\pm 00: 05$ vs 07:36 $\pm 00: 08 ; p<0.001]$.

Moreover, MEQ has an interaction between the three groups ("evening types", "intermediate types", and "morning types") in BT $[F(1344)=14.438 ; p<0.001]$ and WU $[F(1343)=6.015 ; p=0.003]$, greater in the "evening types" group (see Fig. 1a, b). These results were significant after controlling for age and gender.

Table 1 Comparison between pre-health emergency and during health emergency

\begin{tabular}{lccl}
\hline & $\begin{array}{l}\text { Pre-COVID-19 } \\
(\text { mean } \pm \text { SD })\end{array}$ & $\begin{array}{l}\text { During-COVID-19 } \\
(\text { mean } \pm \text { SD })\end{array}$ & $p$ value \\
\hline BT & $23: 43 \pm 1: 06$ & $24: 17 \pm 1: 33$ & $<0.001$ \\
SL & $22.53 \pm 18.61$ & $30.80 \pm 25.34$ & $<0.001$ \\
WU & $7: 47 \pm 1: 15$ & $8: 45 \pm 1: 33$ & $<0.001$ \\
TST & $430.46 \pm 62.18$ & $432.27 \pm 75.37$ & $n s$ \\
TIB & $485.22 \pm 6.46$ & $485.74 \pm 132.46$ & $n s$ \\
PSQI total score & $5.37 \pm 3.01$ & $6.97 \pm 3.54$ & $<0.001$ \\
ISI total score & $9.23 \pm 3.18$ & $10.51 \pm 4.30$ & $<0.001$
\end{tabular}

$B T$ Bed Time (hr); SL Sleep Latency (min); WU Wake-Up (hr); TST Total Sleep Time (min); TIB Time In Bed (min): PSQI score Pittsburgh Sleep Quality Index, total score; ISI score Insomnia Severity Index, total score 
A multivariate significant effect of age was observed on dependent variables in the two groups (students and workers) only during-COVID-19 [Pillai $V=0.048 ; p=0.010$ ]. Concerning univariate $F$ tests on during-COVID-19, we found an effect on BT $[F(1377)=3.695 ; p=0.050 ; \eta 2=0.010]$, and $\mathrm{WU}[F(1377)=9.719 ; p=0.002 ; \eta 2=0.025]$.

In particular, during the lockdown period due to the health emergency, the impact of the delay in BT and in WU was more pronounced in students. Concerning BT, we observed a principal effect of pre- and during-COVID-19 [ $F$ $(1345)=41.420 ; p<0.001]$, a principal effect of employment $[F(1345)=52.216 ; p<0.001]$, and an interaction between the two $[F(1345)=7.248 ; p=0.007]$. Concerning WU, we observed a principal effect of pre- and during-COVID-19 $[F(1345)=41.420 ; p<0.001]$, a principal effect of employment $[F(1345)=52.216 ; p<0.001]$, and an interaction of the two $[F(1345)=7.248 ; p=0.007]$. See Fig. 2a, b.

During COVID-19 emergency, BT was delayed in time of about 16 min for workers and of about 39 min for students $[t(204.282)=-3.2819 ; p=0.001]$, while $\mathrm{WU}$ was delayed in time about $37 \mathrm{~min}$ for workers and $64 \mathrm{~min}$ for students $[t$ $(344)=-2.591 ; p=0.010]$.

Figure 3 shows a significant increase of sleep problems (PSQI $\geq 5$ ) between before and during COVID-19 emergency in both student population (Fig. 3a) (58 vs $73.3 \%)\left[\chi^{2}\right.$
$(1)=25.076 ; p<0.001]$ as well as in workers group (Fig. 3b) (48.2 vs $60.2 \%)\left[\chi^{2}(1)=21.234 ; p<0.001\right]$.

Figure 4 shows that only in the students group (a), a significant increase of clinical insomnia occurred during COVID-19 period $\left[\chi^{2}(1)=13.496 ; p<0.001\right]$, in particular a significant increase of the moderate insomnia category was observed. In the workers group (b), there was only a slight but not significant increase of clinical insomnia.

When we look at which type of insomnia was more prevalent (ISI items) we found that, as expected, almost $40 \%$ of students had at least a mild sleep initiation problem (ISI item 1) already before emergency period that significantly increased to 55\% during COVID-19 (see Fig. 5). This change in sleep initiation is statistically significant both in students (a) $\left[\chi^{2}(1)=43.738 ; p<0.001\right]$ and in workers (b) $\left[\chi^{2}(1)=15.175 ; p<0.001\right]$.

For both maintenance insomnia and early morning awakening (ISI items 2 and 3), 20\% showed problems already before COVID-19 with an increase up to $30 \%$ after COVID19 (see Figs. 6 and 7). This change in sleep maintenance (ISI item 2) is statistically significant both in students (a) $\left[\chi^{2}\right.$ $(1)=43.967 ; p<0.001]$ and in workers $(b)\left[\chi^{2}(1)=29.363\right.$; $p<0.001$ ], as well as in early morning awakening (ISI item 3) [Students (a): $\chi^{2}(1)=37.133 ; p<0.001$ and Workers (b): $\left.\chi^{2}(1)=23.330 ; p<0.001\right]$.
Fig. 1 a Change in BT before and during COVID-19 emergency in different chronotypes; b change in WU before and during COVID-19 emergency in different chronotypes
Fig. 2 a Change in BT before and during COVID-19 emergency in workers and students; b change in WU before and during COVID-19 emergency in workers and students
Bed Time [BT, hr]

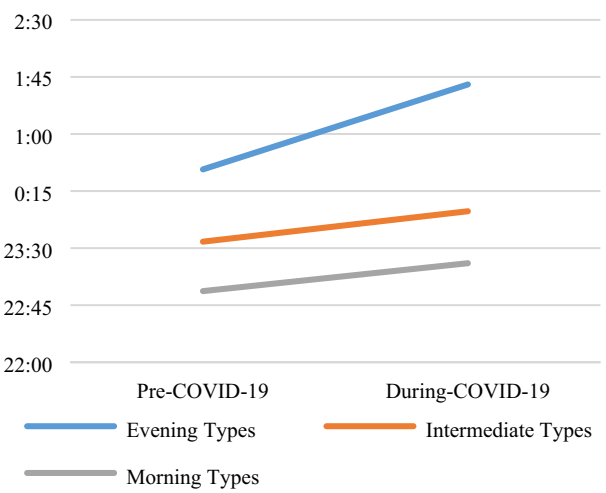

Bed Time [BT, hr]

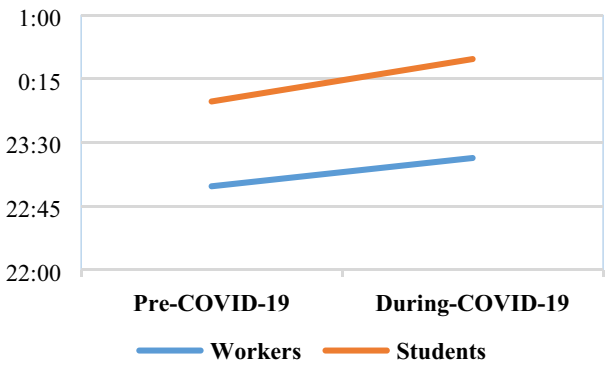

Wake-Up [WU, hr]

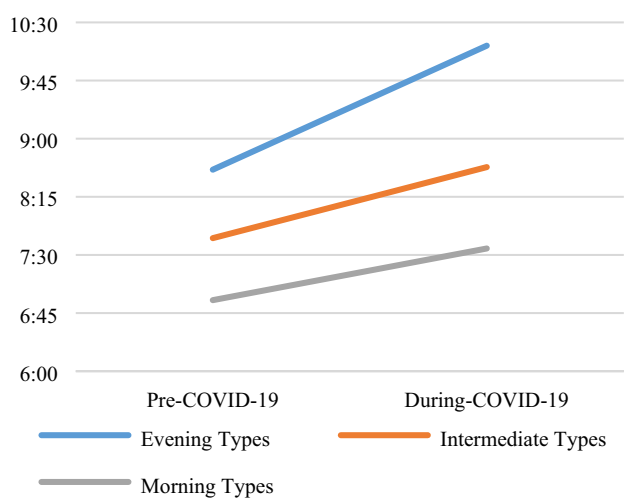

Wake-Up [WU, hr]

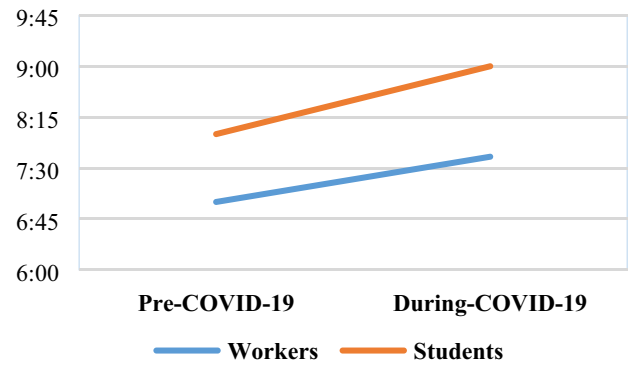




\section{Students PSQI Total score}

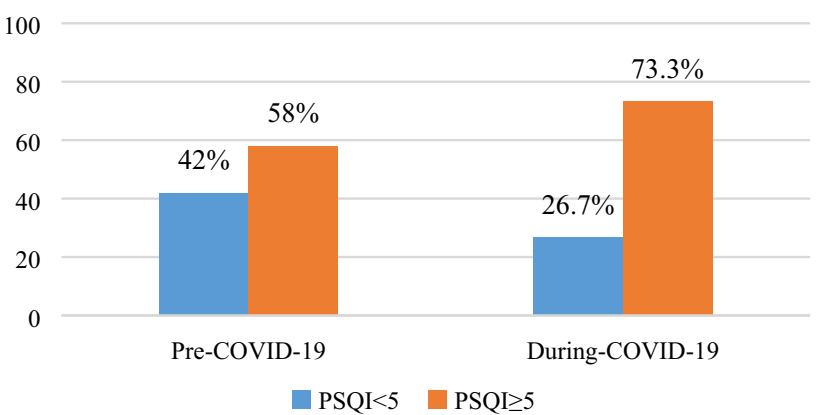

\section{Workers \\ PSQI Total score}

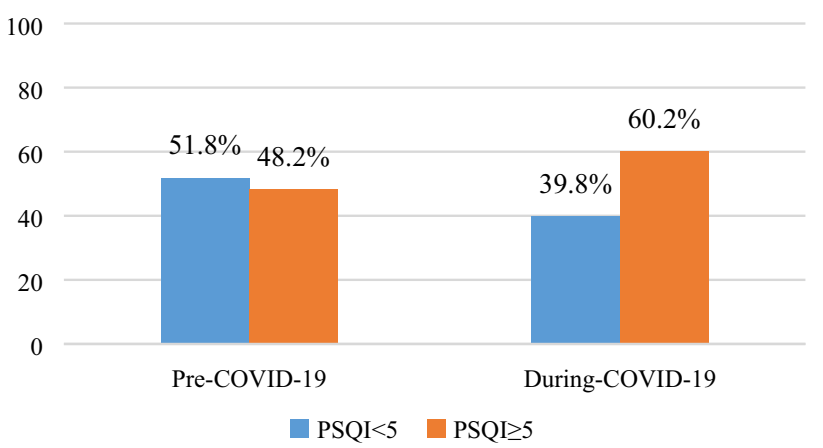

Fig. 3 Percentage of change in PSQI score $(<$ or $\geq 5)$ before and during COVID-19 emergency in students (a) and workers (b)

Moreover, in workers, we observed a prevalence of maintenance insomnia (ISI item 2) before COVID-19 of 24\% that significantly increase during COVID-19 reaching 40\%, while workers with difficulties in sleep initiation (ISI item 1) were only $15 \%$ that dramatically increased to $42 \%$.

In our sample, $27.8 \%$ showed depressive symptoms (between moderate and severe), while $34.3 \%$ showed anxious symptoms (between moderate and severe). In particular, the analysis showed that these symptoms were more frequent in students: clinically significant somatic-affective BDI score was found in $12.9 \%$ of workers and in $25.9 \%$ of students [ $\left.\chi^{2}(1)=6.824 ; p=0.009\right]$, cognitive BDI score in $24.7 \%$ of workers and in $43.2 \%$ of students $\left[\chi^{2}(1)=10.191\right.$; $p=0.001]$, and BAI total score in $21.5 \%$ of workers and in $38.2 \%$ of students $\left[\chi^{2}(1)=8.798 ; p=0.003\right]$.

Correlative analysis in the total sample showed that, during COVID-19 emergency, older subjects had lower TST $(r=-0.232 ; p<0.001)$, but also lower BT $(r=-0.283$; $p<0.001)$, lower WU $(r=-0.369 ; p<0.001)$, and lower ISI score $(r=-0.134 ; p=0.007)$. Moreover, older subjects had lower scores in somatic-affective BDI $(r=-0.268$; $p<0.001)$, cognitive BDI $(r=-0.207 ; p<0.001)$, and
Students

ISI Total score

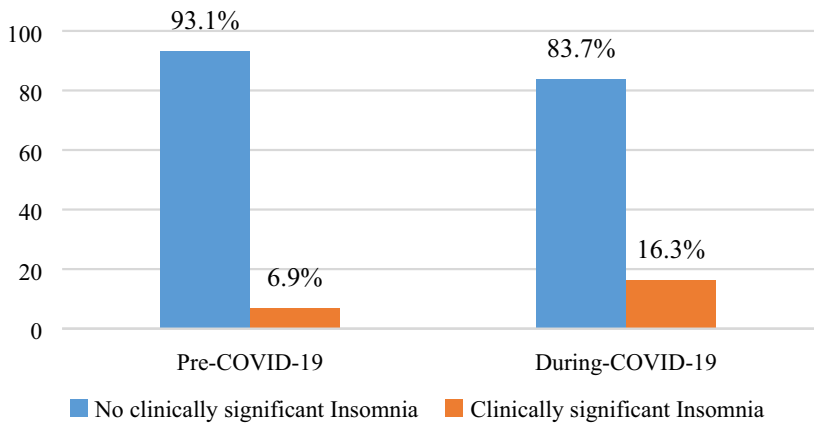

Workers

ISI Total score

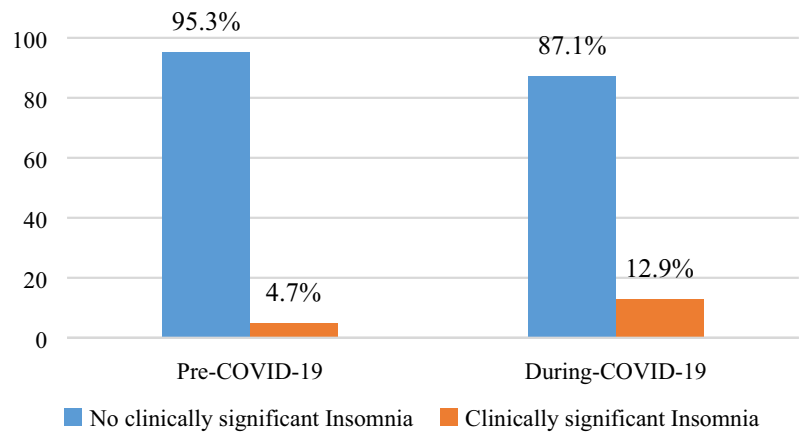

Fig. 4 Percentage of change in ISI scores before and during COVID19 emergency in students (a) and workers (b)

total BDI $(r=-0.174 ; p=0.001)$. PSQI total score during COVID-19 emergency, in the whole sample, directly correlates with BDI total score $(r=0.497 ; p<0.001)$ and BAI total score $(r=0.559 ; p<0.001)$.

We also evaluated the influence of gender. The analysis of the total sample, during COVID-19 emergency, showed that women had worse total scores than men both in PSQI $[t(195.710)=23.251 ; p=0.001]$ and in ISI $[t(177.624)=3.051 ; p=0.003]$. Gender differences in workers showed that female had higher sleep latency $[t(77.093)=2.970 ; p=0.004]$, worse scores in total PSQI $[t(74.715)=3.397 ; p=0.001]$, and in total ISI $[t$ $(71.571)=4.271 ; p<0.001]$ than males. No gender difference was found in students.

\section{Discussion}

Our study shows that the Italian lockdown had a significant impact both on sleep and on psycho-emotional well-being. The impact was greater in students than in administrative staff workers, and in females than in males. In our groups, we observed poor quality of sleep and poor sleep hygiene 


\section{Students}

\section{ISI Item 1}

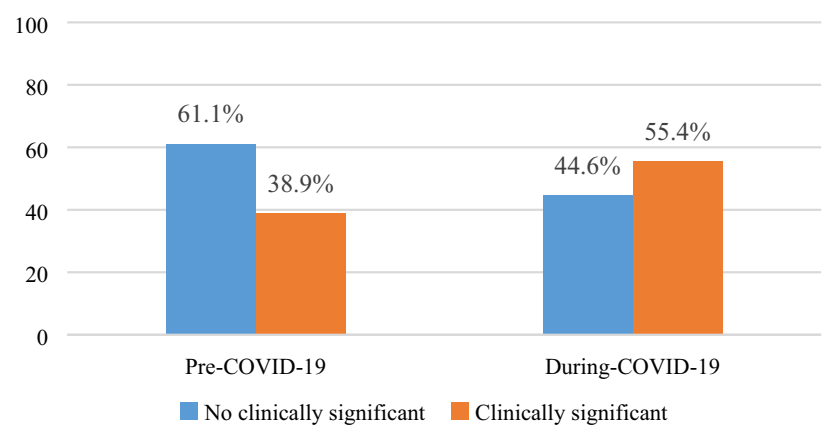

Workers

ISI Item 1

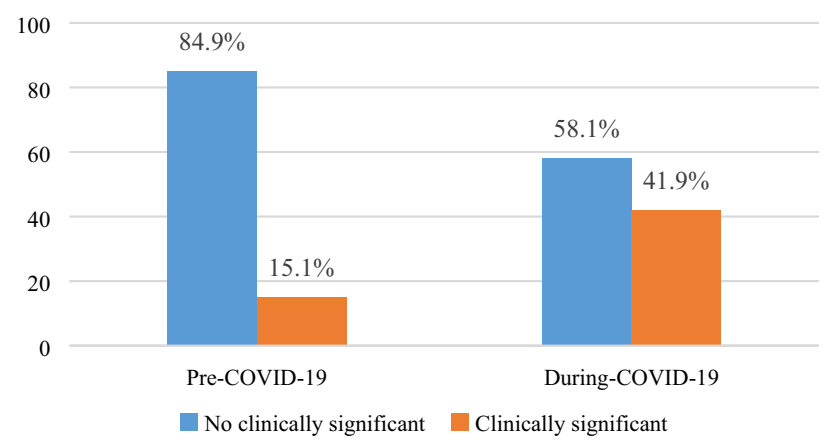

Fig. 5 Percentage of change in ISI Item 1 [sleep initiation], before and during COVID-19 emergency in students (a) and workers (b)

during COVID-19 lockdown, as observed in other studies conducted in the general population $[15,27]$. In particular, a worsening of quality of sleep (according to PSQI) and of symptoms related to insomnia (according to ISI) was found despite no change in TST. However, there was a significant increase in SL (22 vs $30 \mathrm{~min}$ ) that did not reach the clinical significance of greater than $30 \mathrm{~min}$. Moreover, there was an increase of $\mathrm{BT}$ and $\mathrm{WU}$ possibly related to changes in personal lifestyles.

In literature, there are only few findings concerning the effect of social isolation due to COVID-19 on chronotype. Leone and colleagues [28] found a delay in BT and in WU during social isolation, underlying that subjects aligned their sleep timing with their internal time. In our study, we observed a modification in chronotypes between pre- and during-COVID-19, mostly in "evening types". These findings could suggest that, during social isolation without a rigorous schedule, "evening types" go to bed and wake up later, following their biological rhythm, while in "intermediate types" and "morning types" the effects are less, because their biological rhythm is more compliant to social routine.

Our results on ISI items showed that, in students, the sleep initiation problem is the most prevalent before and

\section{Students \\ ISI Item 2}

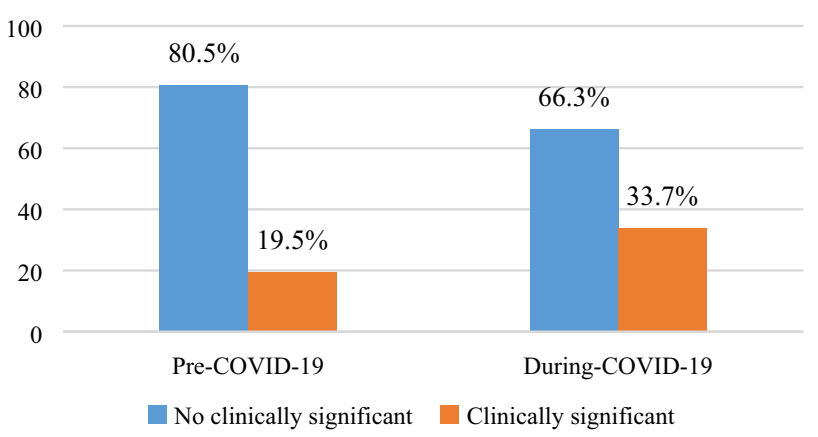

Workers

ISI Item 2

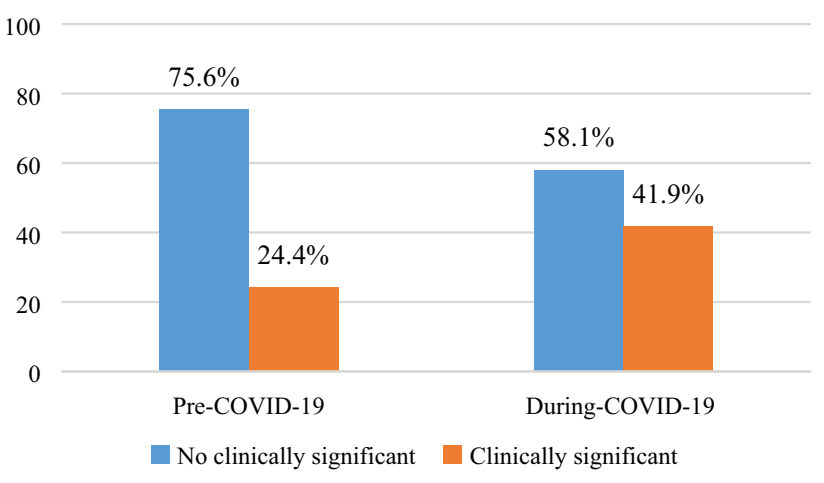

Fig. 6 Percentage of change in ISI Item 2 [sleep maintenance], before and during COVID-19 emergency in students (a) and workers (b)

during COVID-19 (39 vs 55\%). This is of particular interest, because even with the delay of BT and WU time, the increment in time to fall asleep is still a problem in more than half of our young student sample. On the other hand in the workers category, the most frequent sleep difficulty before COVID-19 was the sleep maintenance that was present in $24 \%$ of the sample and that increased to around $40 \%$ during COVID-19. It is interesting to underline the dramatic increase of sleep latency problem from 15 to $42 \%$ of the sample, possibly due to a problem of increased anxiety and worries before sleep in this category of workers.

In a task force work of the European CBT-I Academy, Altena et al. [13] reported that during the lockdown, sleep habits changed due to social restrictions and to a modification of social rhythms, like working and daily activities schedule [14, 29]. In our study, home confinement had a higher impact on sleep habits on students compared to workers in terms of BT and WU delay. BT was delayed of about 16 min for workers and of about 39 min for students, while WU was delayed of about $37 \mathrm{~min}$ in workers and $64 \mathrm{~min}$ for students. Cellini et al. [14] did not find differences in BT 


\section{Students \\ ISI Item 3}

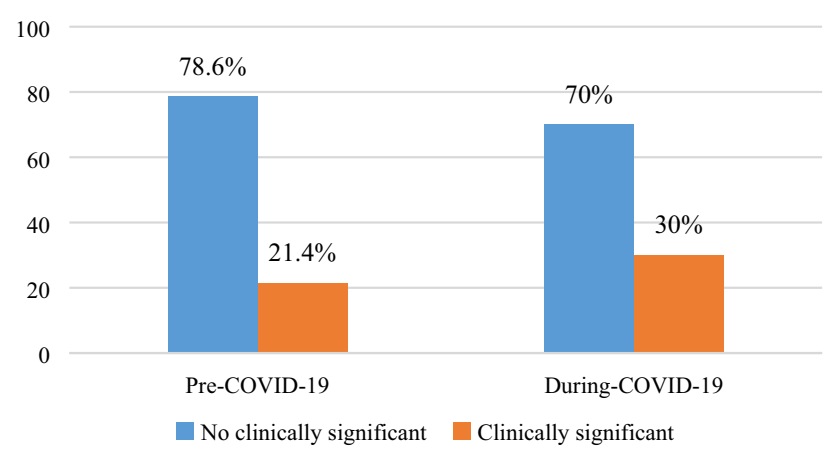

Workers

ISI Item 3

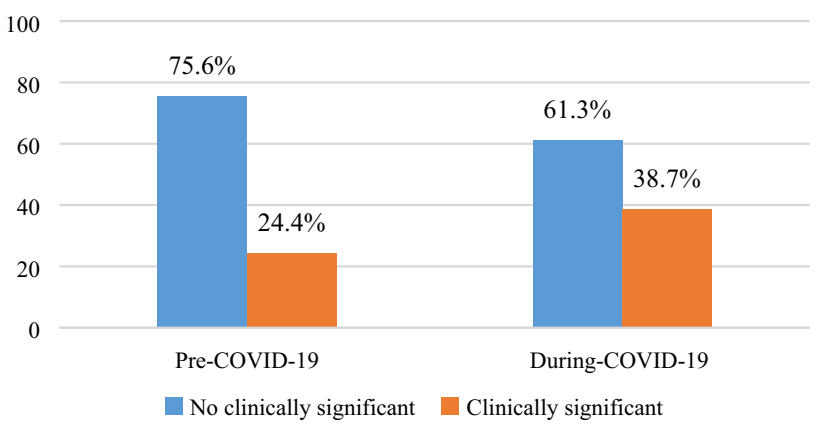

Fig. 7 Percentage of change in ISI Item 3 [early morning awakening], before and during COVID-19 emergency in students (a) and workers (b)

delay between workers and students (about 40 min in both), while WU delay was higher in workers than in students (60 and 13 min, respectively).

Concerning psycho-emotional aspect, $27.8 \%$ of our sample showed depressive symptoms between moderate and severe, while $34.3 \%$ of our sample showed anxious symptoms between moderate and severe. Similar results are reported in the literature about COVID-19 and psychoemotional distress [8-12, 14, 30]. In all these studies and in our sample, psycho-emotional distress has a greater impact on students. In particular, Cellini and colleagues [14] found that in their sample composed by university students and workers, $24.2 \%$ reported depressive symptoms from moderate to severe, and $32.6 \%$ reported anxious symptoms from moderate to severe.

A more marked impact in students than in workers has been observed in our sample, as well as in other studies. The effect of social isolation on feelings of loneliness, of vulnerability and on emergency worry may have led young people to feel less efficient in overcoming the problem, provoking an increase of anxious and depressive symptoms. Anxiety and fear, in addition to provoking a worsening in quality of sleep, increased cortisol levels, reduced melatonin synthesis, with changes in the biological rhythms [31, 32]. Also, a dysregulation of the hypothalamic-pituitary-adrenal axis can be involved in the relationship between poor sleep and feelings of loneliness and fear [27, 33].

However, this study has several limitations. First, the explorative nature of this study is one of the major limitations. Future longitudinal studies are needed to evaluate the residual effects of social isolation after health emergency. Second, we assessed only a restricted cohort of participants (university students and administration staff). For this reason, it is not possible to extend our findings on general population. Third, we used a web-based survey that makes possible selection bias. This survey was composed only by self-reported questionnaires that are not deepened with clinical and instrumental examinations.

The results of our study may provide support for the implementation of some interventions for well-being in pandemic condition. It would be important to offer psychological supports on most vulnerable categories (as women and students), and psycho-educational interventions on sleep and on circadian rhythms to maintain a normal sleep-wake schedule and daily routine during periods of isolation.

\section{Compliance with ethical standards}

Conflicts of interest This work had no financial support. None of the authors of this manuscript had conflicts of interest.

Ethical approval All participants provided written informed consent previously approved by the local ethical committee.

\section{References}

1. Istituto Superiore di Sanità. (2020) Comunicato stampa $\mathrm{N}^{\circ} 19 / 2020$. Studio ISS. Un paziente su cinque positivo al coronavirus ha tra i 19 e i 50 anni. Retrieved from https://www.iss.it/ comunicati-stampa1

2. Bao Y, Sun Y, Meng S, Shi J, Lu L (2020) 2019-nCoV epidemic: address mental health care to empower society. Lancet. https:// doi.org/10.1016/S0140-6736(20)30309-3

3. Hawryluck L, Gold WL, Robinson S, Pogorski S, Galea S, Styra $R$ (2004) SARS control and psychological effects of quarantine, Toronto, Canada. Emerg Infect Dis. https://doi.org/10.3201/eid10 07.030703

4. Wu KK, Chan SK, Ma TM (2005) Posttraumatic stress, anxiety, and depression in survivors of severe acute respiratory syndrome (SARS). J Trauma Stress. https://doi.org/10.1002/jts.20004

5. Wu KK, Chan SK, Ma TM (2005) Posttraumatic stress after SARS. Emerg Infect Dis. https://doi.org/10.3201/eid1108.041083

6. Mak IW, Chu CM, Pan PC, Yiu MG, Ho SC, Chan VL (2010) Risk factors for chronic post-traumatic stress disorder (PTSD) in SARS survivors. Gen Hosp Psychiatry. https://doi.org/10.1016/j. genhosppsych.2010.07.007

7. Brooks SK, Webster RK, Smith LE, Woodland L, Wessely S, Greenberg N, Rubin GJ (2020) The psychological impact of 
quarantine and how to reduce it: rapid review of the evidence. Lancet. https://doi.org/10.1016/S0140-6736(20)30460-8

8. Wang C, Pan R, Wan X, Tan Y, Xu L, Ho CS, Ho RC (2020) Immediate psychological responses and associated factors during the initial stage of the 2019 Coronavirus disease (COVID-19) epidemic among the general population in China. Int J Environ Res Public Health. https://doi.org/10.3390/ijerph17051729

9. Xiao H, Zhang Y, Kong D, Li S, Yang N (2020) The effects of social support on sleep quality of medical staff treating patients with coronavirus disease 2019 (COVID-19) in January and February 2020 in China. Med Sci Monit. https://doi.org/10.12659/ MSM.923549

10. Xiao H, Zhang Y, Kong D, Li S, Yang N (2020) Social capital and sleep quality in individuals who self-isolated for 14 days during the coronavirus disease 2019 (COVID-19) Outbreak in January 2020 in China. Med Sci Monit. https://doi.org/10.12659 /MSM.923921

11. Li Z, Ge J, Yang M, Feng J, Qiao M, Jiang R, Bi J, Zhan G, Xu X, Wang L, Zhou Q, Zhou C, Pan Y, Liu S, Zhang H, Yang J, Zhu B, Hu Y, Hashimoto K, Jia Y, Wang H, Wang R, Liu C, Yang C (2020) Vicarious traumatization in the general public, members, and non-members of medical teams aiding in COVID-19 control. Brain Behav Immun. https://doi.org/10.1016/j.bbi.2020.03.007

12. Huang Y, Zhao N (2020) Generalized anxiety disorder, depressive symptoms and sleep quality during COVID-19 outbreak in China: a web-based cross-sectional survey. Psychiatry Res. https://doi. org/10.1016/j.psychres.2020.112954

13. Altena E, Baglioni C, Espie CA, Ellis J, Gavriloff D, Holzinger B, Schlarb A, Frase L, Jernelöv S, Riemann D (2020) Dealing with sleep problems during home confinement due to the COVID19 outbreak: practical recommendations from a task force of the European CBT-I Academy. J Sleep Res. https://doi.org/10.1111/ jsr. 13052

14. Cellini N, Canale N, Mioni G, Costa S (2020) Changes in sleep pattern, sense of time and digital media use during COVID-19 lockdown in Italy. J Sleep Res. https://doi.org/10.1111/jsr.13074

15. Li Y, Qin Q, Sun Q, Sanford LD, Vgontzas AN, Tang X (2020) Insomnia and psychological reactions during the COVID-19 outbreak in China. J Clin Sleep Med. https://doi.org/10.5664/ jcsm. 8524

16. Buysse DJ, Reynolds CF, Monk TH, Berman SR, Kupfer DJ (1989) The pittsburgh sleep quality index: a new instrument for psychiatric practice and research. Psychiat Res. https://doi. org/10.1016/0165-1781(89)90047-4

17. Curcio G, Tempesta D, Scarlata S, Marzano C, Moroni F, Rossini PM, Ferrara M, De Gennaro L (2013) Validity of the Italian version of the Pittsburgh Sleep Quality Index (PSQI). Neurol Sci. https://doi.org/10.1007/s10072-012-1085-y

18. Morin CM, Belleville G, Be'langer L, Ivers H (2011) The insomnia severity index: psychometric indicators to detect insomnia cases and evaluate treatment response. Sleep. https://doi. org/10.1093/sleep/34.5.601

19. Castronovo V, Galbiati A, Marelli S, Brombin C, Cugnata F, Giarolli L, Anelli MM, Rinaldi F, Ferini-Strambi L (2016) Validation study of the Italian version of the Insomnia Severity Index (ISI). Neurol Sci. https://doi.org/10.1007/s10072-016-2620-z
20. Horne JA, Ostberg O (1976) A self-assessment questionnaire to determine morningness-eveningness in human circadian rhythms. Int J Chronobiol 4(2):97-110

21. Natale V, Esposito MJ, Martoni M, Fabbri M (2006) Validity of the reduced version of the morningness eveningness questionnaire. Sleep Biol Rhythms. https://doi.org/10.111 1/j.1479-8425.2006.00192.x

22. Taillard J, Philip P, Chastang JF, Bioulac B (2004) Validation of Horne and Ostberg morningness-eveningness questionnaire in a middle-aged population of French workers. J Biol Rhythms. https ://doi.org/10.1177/0748730403259849

23. Beck AT, Epstein N, Brown G, Steer RA (1988) An inventory for measuring clinical anxiety: psychometric properties. J Consult Clin Psychol. https://doi.org/10.1037//0022-006x.56.6.893

24. Sica C, Coradeschi D, Ghisi M, Sanavio E (2006) Beck anxiety inventory. Manuale. Organizzazioni Speciali, Adattamento italiano, Firenze

25. Beck AT, Steer RA, Brown GK (1996) Beck depression inventoryII. San Antonio 78(2):490-498

26. Ghisi M, Flebus GB, Montano A, Sanavio E, Sica C (2006) Beck depression inventory. Manuale. Organizzazioni Speciali, Adattamento italiano, Firenze

27. Voitsidis P, Gliatas I, Bairachtari V, Papadopoulou K, Papageorgiou G, Parlapani E, Syngelakis M, Holeva V, Diakogiannis I (2020) Insomnia during the COVID-19 pandemic in a Greek population. Psychiatry Res. https://doi.org/10.1016/j.psych res.2020.113076

28. Leone MJ, Sigman M, Golombek D (2020) Effects of social isolation on human sleep and chronotype during the COVID-19 pandemic. Available at SSRN: https://ssrn.com/abstract $=3624469$ or http://dx.doi.org/10.2139/ssrn.3624469

29. Losada-Baltar A, Jiménez-Gonzalo L, Gallego-Alberto L, Pedroso-Chaparro MDS, Fernandes-Pires J, Márquez-González M (2020) "We're staying at home". Association of self-perceptions of aging, personal and family resources and loneliness with psychological distress during the lockdown period of COVID-19. J Gerontol B Psychol Sci Soc Sci. https://doi.org/10.1093/geronb/ gbaa048

30. Huang Y, Zhao N (2020) Chinese mental health burden during the COVID-19 pandemic. Asian J Psychiatr. https://doi.org/10.1016/j. ajp.2020.102052

31. van Santen A, Vreeburg SA, Van der Does AJ, Spinhoven P, Zitman FG, Penninx BW (2011) Psychological traits and the cortisol awakening response: results from the Netherlands study of depression and anxiety. Psychoneuroendocrinology. https://doi. org/10.1016/j.psyneuen.2010.07.014

32. Cardinali DP, Srinivasan V, Brzezinski A, Brown GM (2012) Melatonin and its analogs in insomnia and depression. J Pineal Res. https://doi.org/10.1111/j.1600-079X.2011.00962.x

33. Griffin SC, Williams AB, Mladen SN, Perrin PB, Dzierzewski JM, Rybarczyk BD (2019) Reciprocal effects between loneliness and sleep disturbance in older Americans. J Aging Health. https ://doi.org/10.1177/0898264319894486 (published online ahead of print, 2019 Dec 21) 